\title{
Milk Fat Globule-Epidermal Growth Factor VIII Amelio- rates Brain Injury in the Subacute Phase of Cerebral Isch- emia in an Animal Model
}

\author{
Jong-Il Choi, ${ }^{1}$ Ho-Young Kang, ${ }^{2}$ Choongseong Han, ${ }^{3}$ Dong-Hun Woo, ${ }^{3}$ Jong-Hoon Kim, ${ }^{4}$ Dong-Hyuk Park ${ }^{2,5}$ \\ Department of Neurosurgery, Hallym University Kangnam Sacred Heart Hospital, Hallym University College of Medicine, Seoul, Korea \\ Department of Neurosurgery, ${ }^{2}$ Anam Hospital, College of Medicine, Korea University, Seoul, Korea \\ NEXEL Co., Ltd., ${ }^{3}$ Seoul, Korea \\ Laboratory of Stem Cells and Tissue Regeneration, ${ }^{4}$ Department of Biotechnology, College of Life Sciences and Biotechnology, Korea Univer- \\ sity, Seoul, Korea \\ Center of Innovative Cell Therapy and Research, ${ }^{5}$ Anam Hospital, College of Medicine, Korea University, Seoul, Korea
}

Objective : Milk fat globule-epidermal growth factor VIII (MFG-E8) may play a key role in inflammatory responses and has the potential to function as a neuroprotective agent for ameliorating brain injury in cerebral infarction. This study aimed to determine the role of MFG-E8 in brain injury in the subacute phase of cerebral ischemia in a rat model.

Methods : Focal cerebral ischemia was induced in rats by occluding the middle cerebral artery with the modified intraluminal filament technique. Twenty-four hours after ischemia induction, rats were randomly assigned to two groups and treated with either recombinant human MFG-E8 or saline. Functional outcomes were assessed using the modified Neurological Severity Score (mNSS), and infarct volumes were evaluated using histology. Anti-inflammation, angiogenesis, and neurogenesis were assessed using immunohistochemistry with antibodies against ionized calcium-binding adapter molecule 1 (Iba-1), rat endothelial cell antigen-1 (RECA-1), and bromodeoxyuridine (BrdU)/doublecortin (DCX), respectively.

Results : Our results showed that intravenous MFG-E8 treatment did not reduce the infarct volume; however, the mNSS test revealed that neurobehavioral deficits were significantly improved in the MFG-E8-treated group than in the vehicle group. Immunofluorescence staining revealed a significantly lower number of Iba-1-positive cells and higher number of RECA-1 in the periinfarcted brain region, and significantly higher numbers of BrdU- and DCX-positive cells in the subventricular zone in the MFG-E8treated group than in the vehicle group.

Conclusion : Our findings suggest that MFG-E8 improves neurological function by suppressing inflammation and enhancing angiogenesis and neuronal proliferation in the subacute phase of cerebral infarction.

Key Words : Angiogenesis - Inflammation · MFG-E8 - Neurobehavioral outcome · Neuronal proliferation - Subacute cerebral infarction.

\footnotetext{
- Received : September 11, 2019 •Revised : November 11, 2019 •Accepted : December 1, 2019

- Address for reprints : Dong-Hyuk Park

Department of Neurosurgery, Anam Hospital, Korea University Medical Center, College of Medicine, Korea University, 73 Goryeodae-ro, Seongbuk-gu, Seoul 02841, Korea Tel : +82-2-920-6833, Fax : +82-2-929-0629, E-mail : doctorns@korea.com, ORCID : https://orcid.org/0000-0002-4133-9479
} 


\section{INTRODUCTION}

Cerebral infarction is characterized by pan-necrosis in not only neurons but also glia and vascular elements ${ }^{22)}$. At the cerebral hypoperfusion stage, the pathophysiology of cerebral infarction is related to primary anoxic-ischemic cell death and secondary neuronal injury.

Secondary neuronal injury is characterized by the activation of inflammatory cytokines and production of free radicals, thereby leading to the production of potentially toxic materials and apoptotic cells ${ }^{1,3)}$. Therefore, efficient removal of toxic and apoptotic materials is of primary importance for protecting the surrounding tissues from secondary neuronal injury ${ }^{16)}$.

Milk fat globule- epidermal growth factor VIII (MFG-E8) is a $66-\mathrm{kDa}$ glycoprotein originally discovered in mouse milk and mammary epithelium. MFG-E8 was subsequently found to be widely distributed in various tissues in mice and other mammalian species, including humans ${ }^{8,9,26)}$.

MFG-E8, also known as lactadherin, has been demonstrated to act as an intermediary between apoptotic cells in the tissue and phagocytes, such as macrophages, and plays an important role in the inflammatory response and the clearance of apoptotic cells ${ }^{25)}$. Notably, MFG-E8 has recently been recognized as a critical regulator of the inflammatory response, and many studies using various cell and animal models are investigating the potential role of MFG-E8 in secondary neuronal injury in cerebral infarction ${ }^{5)}$.

In acute cerebral infarction, therapies such as intravenous administration of recombinant tissue plasminogen activator within 4.5 hours and mechanical thrombectomy within 24 hours of stroke onset are widely used clinically ${ }^{13)}$. However, after the acute phase of cerebral infarction there is no effective treatment other than supportive medication such as antiplatelet agents.

Therefore, we investigated whether MFG-E8 attenuates the cellular and biochemical toxic responses and enhances neuronal proliferation and angiogenesis after cerebral ischemia, and whether MFG-E8 ameliorates brain injury in the subacute stage of cerebral ischemia in a rat model.

\section{MATERIALS AND METHODS}

\section{Animal model of ischemic stroke}

All animal experiments were approved by the Institutional Review Board of the Korea University (Seoul, Korea; KUIACUC-2016-0253) and were conducted in accordance with the National Institutes of Health Guide for the Care and Use of Laboratory Animals (National Institutes of Health [NIH] publication No. 80-23, revised in 1996). Adult male SpragueDawley rats $(\mathrm{n}=12)$, weighing 300-320 g (Orient Bio Inc., Seongnam, Korea), were used in this study.

Acute stroke was induced using the standard middle cerebral artery occlusion (MCAO) method, as previously described $^{2)}$. Animals were anesthetized with 5\% isoflurane and were maintained under anesthesia with $1.5 \%$ isoflurane in a mixture of $70 \% \mathrm{~N}_{2} \mathrm{O}$ and $30 \% \mathrm{O}_{2}$. After exposure of the common carotid artery, a 4-0 silicone-coated monofilament (Doccol Corp., Sharon, MA, USA) was inserted into the internal carotid artery to occlude the ipsilateral middle cerebral artery. The occlusion was maintained for 90 minutes, and the monofilament was subsequently removed. The animal was tested for spontaneous circling, and the tail-lifting test was also performed. In this experiment, 24 hours after onset of cerebral infarction was defined as subacute stage cerebral infarction.

\section{Experimental groups}

Twenty-four hours after MCAO reperfusion, animals were randomly assigned to one of the following two groups. Rats in the first group $(n=6)$ were administered once recombinant human MFG-E8 (rhMFG-E8; R\&D systems, Minneapolis, MN, USA; Catalog No., 2767-MF; $160 \mu \mathrm{g} / \mathrm{kg} / \mathrm{mL}$ ) intravenously via the tail vein, and those in the vehicle group were administered normal saline $(n=6)$.

\section{Neurobehavioral tests}

Neurobehavioral functions were evaluated on days 1, 4, 8, 11, and 15 after MCAO using the modified Neurological Severity Score (mNSS) ${ }^{4}$. The mNSS is a composite of motor (muscle status and abnormal movement), sensory (visual, tactile, and proprioceptive), and balance tests. Neurological function was graded on a scale from 0 to 18 (normal to maximum deficit). 


\section{Tissue preparation and immunohistochemistry}

Fifteen days after MCAO, the animals were anesthetized with Zoletil $50(30 \mathrm{mg} / \mathrm{kg})$ and xylazine $(5 \mathrm{mg} / \mathrm{kg})$ and were perfused transcardially with saline followed by $4 \%$ paraformaldehyde in $0.1 \mathrm{~mol} / \mathrm{L}$ phosphate buffer. After perfusion, brains were removed and fixed in a $4 \%$ paraformaldehyde solution overnight at $4^{\circ} \mathrm{C}$, and they were then placed in a $30 \%$ sucrose solution for cryoprotection. Frozen brains were sliced into 20- $\mu$ m-thick coronal sections using a cryostat vibratome (CM3050S; Leica Microsystems, Wetzlar, Germany), and these sections were stored at $-80^{\circ} \mathrm{C}$ until further processing.

For immunohistochemistry, tissue sections were treated with $2 \mathrm{~N} \mathrm{HCl}$ for 30 minutes at $37^{\circ} \mathrm{C}$, followed by neutralization with immersion in $0.1 \mathrm{~mol} / \mathrm{L}$ borate buffer ( $\mathrm{pH} 8.5$ ). Nonspecific binding was blocked by incubating sections with $10 \%$ horse serum in phosphate-buffered saline. Sections were then incubated in primary antibodies overnight at $4{ }^{\circ} \mathrm{C}$.

The primary antibodies and dilutions used in immunohistochemistry were as follows : ionized calcium-binding adapter molecule 1 (Iba-1; 1 : 400; Wako Chemicals USA, Inc., Dallas, TX, USA), rat endothelial cell antigen-1 (RECA-1; 1 : 400; BioRad Laboratories, Hercules, CA, USA), bromodeoxyuridine (BrdU; 1 : 50; Roche, Basel, Switzerland), and doublecortin (DCX; 1 : 100; Santa Cruz Biotechnology Inc., Dallas, TX, USA). The following secondary antibodies were used: Alexa Fluor 488 anti-mouse IgG (1 : 400; Invitrogen, Waltham, MA, USA), Alexa Fluor 594 anti-goat IgG (1 : 800; Invitrogen), Alexa Fluor 488 anti-rabbit IgG (1 : 400, Invitrogen), and Rhodamine Red-X-conjugated anti-Mouse IgG (1 : 100; Jackson ImmunoResearch Inc., Burlingame, CA, USA). Staining was visualized by a fluorescent microscope (BX61; Olympus Corp., Tokyo, Japan). Immunofluorescence images were acquired using a Zeiss LSM 700 confocal laser microscope (Carl Zeiss, Oberkochen, Germany).

\section{Measurement of cerebral infarction volume}

For infarct volume measurement, brain sections were stained with $0.1 \%$ cresyl violet (Nissl). Images were acquired using a $4 \times$ objective lens under a bright-field microscope (BF53; Olympus). The areas of infarction were quantified using ImageJ software (NIH, Bethesda, MD, USA), and the infarct volume was calculated by multiplying the sum of the infarct area by the distance between sections. After correcting for edema, the infarction volume was calculated as uni follows :
Corrected Infarct Volume $\%=[$ contralateral hemisphere volume - (ipsilateral hemisphere volume - infarct volume)] / contralateral hemisphere volume $\times 100$.

\section{Quantification and statistics}

To count the total number of BrdU- and DCX-positive cells, six sections were obtained at every $280 \mu \mathrm{m}$, beginning with a section $1.2 \mathrm{~mm}$ rostral to the bregma, and immunohistochemistry and quantification analysis in the subventricular zone (SVZ) were performed to confirm neurogenesis. Iba1-positive cells were counted under a fluorescent microscope (BX61; Olympus) in three microscopic fields of the peri-infarct area of each section. RECA-1 positive vessel density was evaluated in images captured from ischemic hemispheres using MetaMorph imaging software (version 7.8.1; Molecular Devices, San Jose, CA, USA). The sum of the values from all six sections was used as the final value.
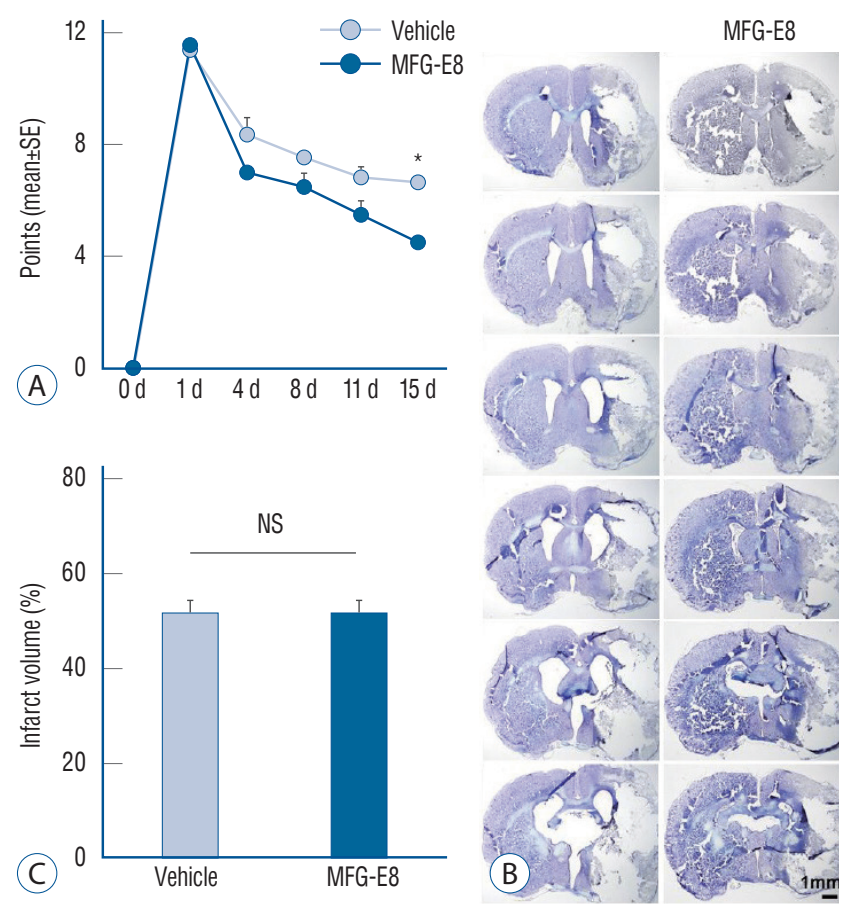

Fig. 1. Modified Neurological Severity Scores and infarct volumes. A and $C$ : Quantification of neurological scores and infarct volumes of vehicleand MFG-E8-treated rats after transient middle cerebral artery occlusion (tMCAO). Neurobehavioral function improved in the MFG-E8-treated group on day 15 after tMCAO. No significant differences in percentage infarct volumes were found between groups. B : Representative images of cresyl violet-stained coronal brain sections 15 days after tMCAO. Scale bar $=1 \mathrm{~mm} .{ }^{*} p<0.001, \mathrm{n}=4-5$ per group. $\mathrm{SE}$ : standard error, MFG-E8 : milk fat globule-epidermal growth factor VIII, NS : no significance. 
All data are presented as mean \pm standard error of the mean. Parametric data were analyzed using one-way analysis of variance, and non-parametric data were analyzed using the Kruskal-Wallis test on ranks, followed by a Mann-Whitney U test. Differences with a $p$-value $<0.05$ were deemed statistically significant.

\section{RESULTS}

\section{Effects of MFG-E8 on neurobehavioral outcomes}

Using the ischemic stroke model, we evaluated the effect of MFG-E8 treatment on the recovery of behavioral functions using the mNSS test. Before MCAO, neurological scores were similar among the experimental and control groups (Fig. 1A). On day 1 after stroke, no treatment $\times$ time interactions were detected for any of the mNSS scores, and no significant differences in functional recovery were found between groups. However, as shown in Fig. 1A, lower mNSS mean scores were observed on days 4, 8, 11, and 15 after stroke in the MFG-E8treated group than in the vehicle group $(p<0.05)$, indicating significantly improved neurobehavioral outcomes in MFG-E8-treated rats.

\section{Cerebral infarction volume}

Infarcts were found in the cerebral hemisphere ipsilateral to the occlusion in all rats (Fig. $1 \mathrm{~B}$ and $\mathrm{C}$ ). The average infarct volumes were $50.6 \pm 2.31 \mathrm{~mm}^{3}$ and $51.3 \pm 7.43 \mathrm{~mm}^{3}$ in the vehicle and MFG-E8-treated groups, respectively. No significant differences in infarct volumes were found between these two groups ( $p>0.05)$.

\section{Immunohistochemical analyses}

MFG-E8 inhibits microglial activation in ischemic hemispheres

Immunohistochemical analyses revealed a lower number of Iba-1-positive cells (Fig. 2A) in the peri-infarcted area in the MFG-E8-treated group than in the vehicle group. A significantly lower percentage of hypertrophic or amoeboid Iba1-positive cells was also found in the MFG-E8-treated group than in the vehicle group ( $p<0.001$; Fig. $2 \mathrm{~B}$ ). These findings indicate that MCAO-induced accumulation of microglia-positive cells in ischemic brain regions is attenuated by intravenous MFG-E8 treatment, and MFG-E8 suppresses inflammation during the subacute stage of cerebral ischemia.
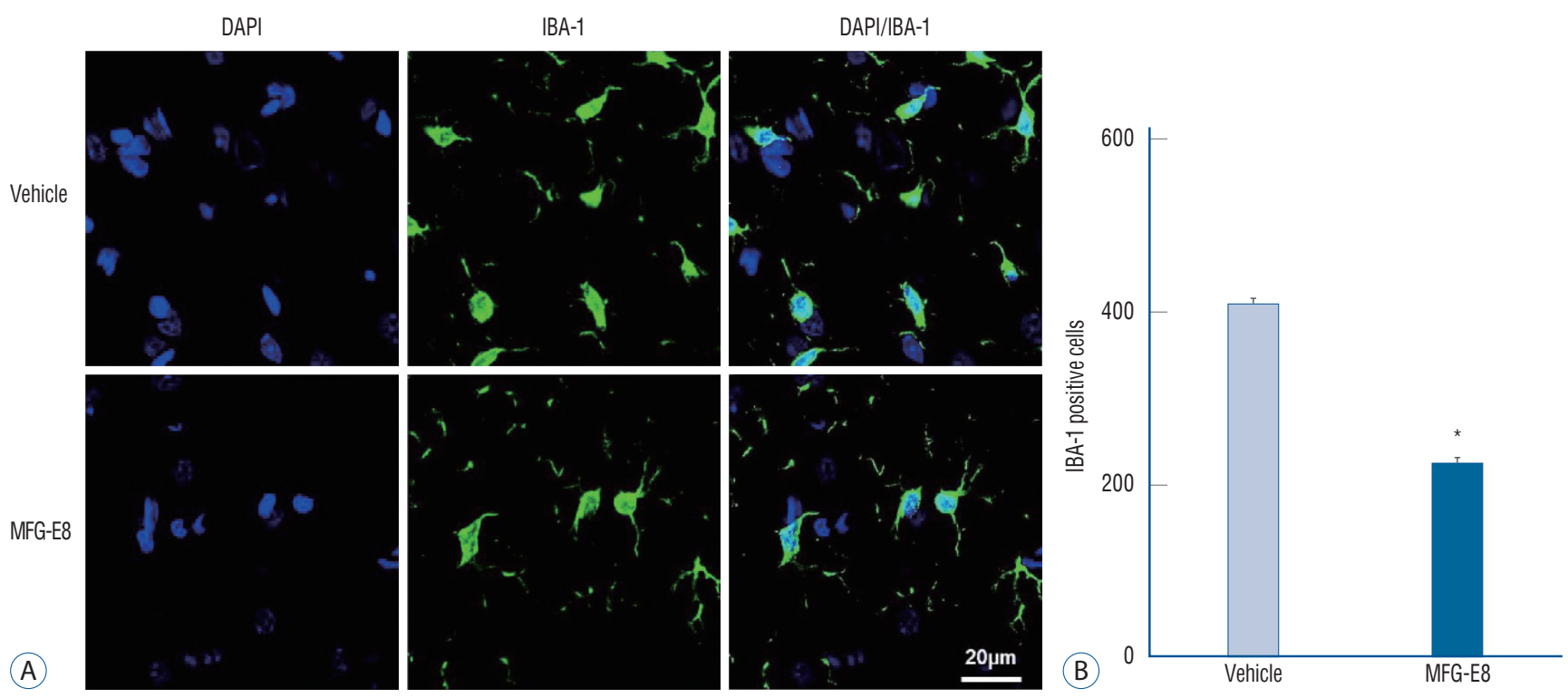

Fig. 2. MFG-E8 inhibits microglial activation. A : Representative images of Iba-1 immunoreactivity counterstained with DAPI evaluated in ischemic hemispheres on day 15 after ischemia induction. Scale bar=20 mm. B : Quantification of the number of microglia found in the peri-infarct area. Iba1-positive cells (green) decreased in the MFG-E8-treated group compared with the vehicle-treated group. Data are expressed as mean \pm SEM. ${ }^{*} p<0.001, \mathrm{compa}$ red with the vehicle-treated group by Student's t-test. DAPI : 4,6-diamidino-2-phenylindole, Iba-1 : ionized calcium-binding adapter molecule 1, MFG-E8 : milk fat globule-epidermal growth factor VIII, SEM : standard error of mean. 
MFG-E8 enhances angiogenesis in the peri-infarct area in the rat brain

Immunofluorescence staining analyses found a significantly higher number of RECA-1-positive cells in the peri-infarcted area in the MFG-E8-treated group than in the vehicle group $(p<0.05)$ (Fig. 3). The findings support the hypothesis that intravenous MFG-E8 treatment promotes angiogenesis in the peri-infarct area after the subacute stage of cerebral ischemia.
Vehicle

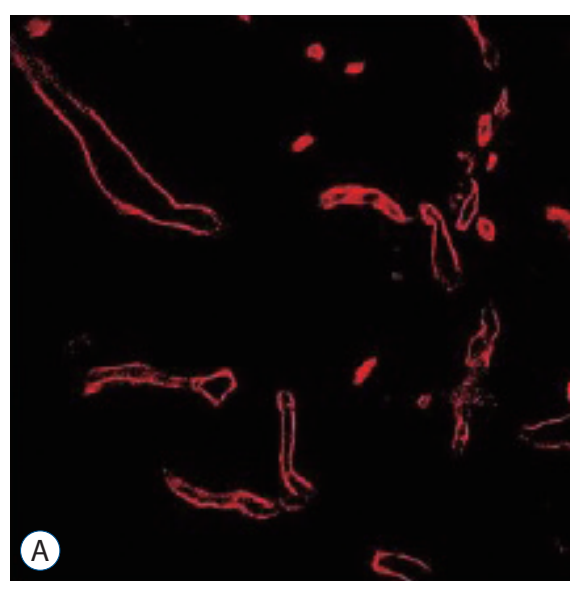

MFG-E8

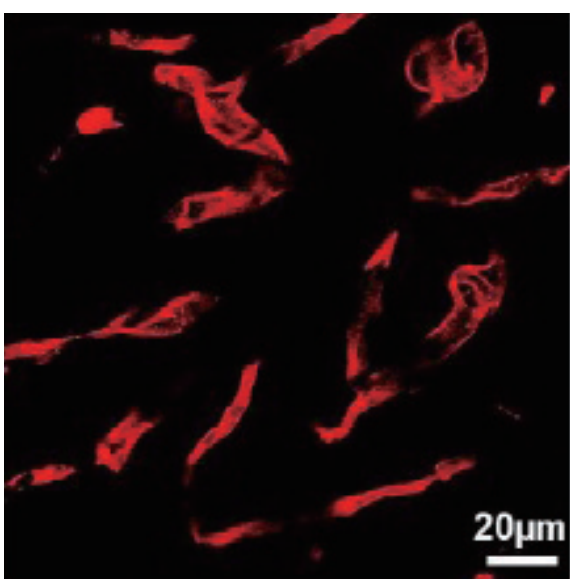

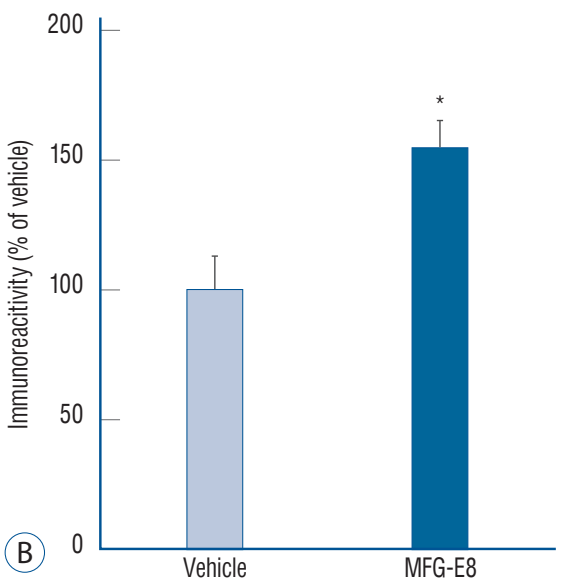

Fig. 3. MFG-E8 enhances angiogenesis in the peri-infarct area in ischemic rat brains. A : Representative images of RECA-1-positive microvessels in ischemic hemispheres on day 15 after ischemia induction. Scale bar $=20 \mathrm{~mm}$. B : Quantitative analysis of RECA-1 immunoreactivity determined from 6 cryosections ( $n=4-5$ per group). RECA-1 (a marker of endothelial cells) immunostaining shows an increased area of vessels in the MFG-E8-treated group. Data are expressed as percentages of immunoreactivity in MFG-E8-treated stroke brains compared with vehicle-treated stroke brains. ${ }^{*} p<0.05$, compared with the vehicle-treated group by Student's t-test. MFG-E8 : milk fat globule-epidermal growth factor VIII, RECA-1 : rat endothelial cell antigen-1.
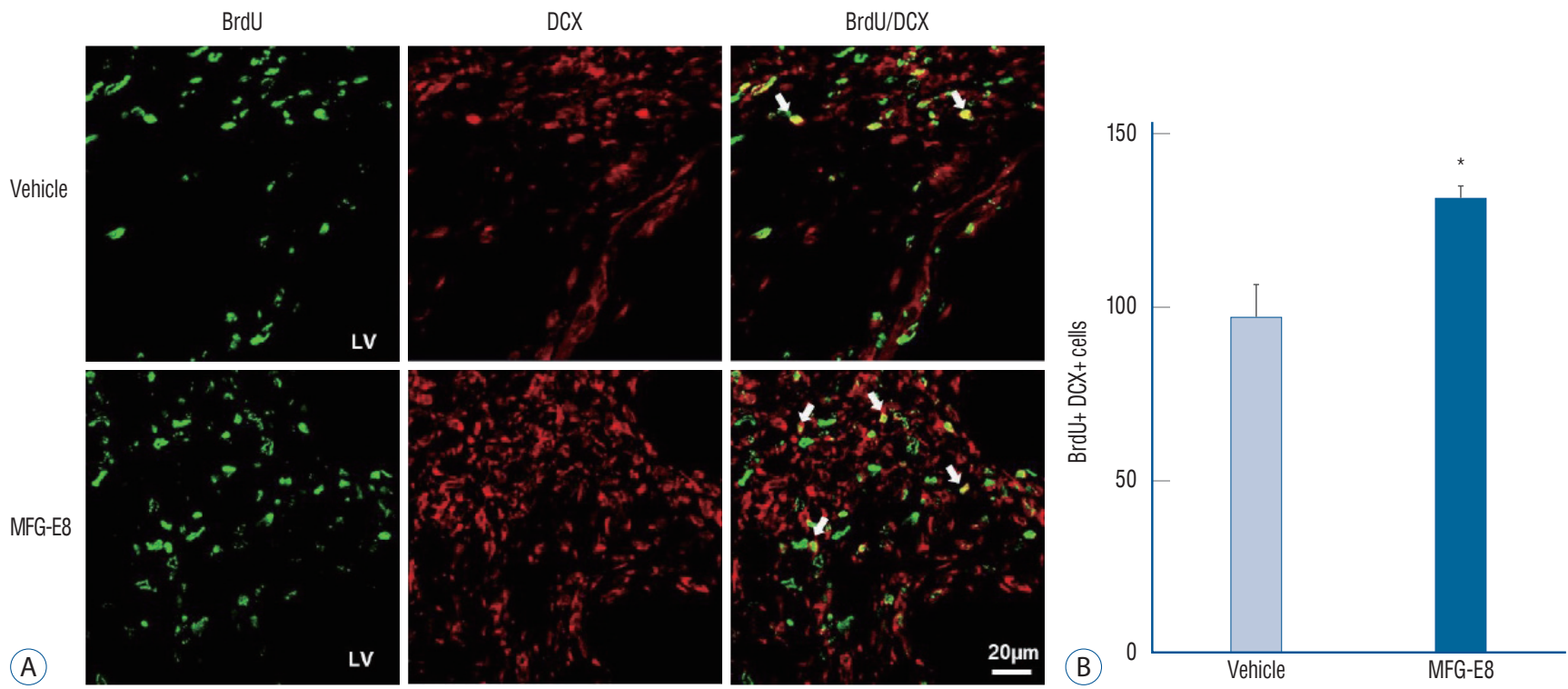

Fig. 4. MFG-E8 promotes neurogenesis in ischemic rat brains. Effects of MFG-E8 treatment on neurogenesis were examined by immunostaining of BrdU (green) and DCX (red). A : BrdU/DCX double-positive cells in the SVZ on day 15 after stroke in the rat brain. Arrows indicate BrdU/DCX double-labeled cells. Scale bar $=20 \mathrm{~mm}$. B : The total number of BrdU/DCX double-positive cells in six sections of each animal was counted ( $\mathrm{n}=4-5 \mathrm{per}$ group). The number of BrdU/DCX double-positive cells was higher in the MFG-E8 treatment group than in the vehicle-treated group. Data are expressed as mean \pm SEM. ${ }^{*} p<0.001$, compared with the vehicle-treated group by Student's t-test. BrdU : bromodeoxyuridine, DCX : doublecortin, MFG-E8 : milk fat globuleepidermal growth factor VIII, LV : lateral ventricle, SVZ : subventricular zone, SEM : standard error of mean. 


\section{MFG-E8 promotes neurogenesis in the SVZ}

Significantly increased BrdU- and DCX-positive cells were found in the SVZ in the MFG-E8 treatment group than in the vehicle treatment group $(p<0.001)$ (Fig. 4$)$. The findings indicate that intravenous MFG-E8 treatment promotes neurogenesis in the injured brain regions after the subacute stage of cerebral ischemia.

There were a significantly lower number of Iba-1-positive cells and higher number of RECA-1 in the peri-infarcted brain region, as well as significantly higher numbers of BrdU- and DCX-positive cells in the subventricular region in the MFG-E8treated group than in the vehicle group.

\section{DISCUSSION}

Our study showed that intravenous MFG-E8 treatment significantly improved neurobehavioral deficits after cerebral ischemia, although it did not have an impact on the infarct volume. Additionally, immunofluorescence staining revealed that MFG-E8-treated animals had a significantly lower number of Iba-1-positive cells and higher number of RECA-1 in the periinfarcted brain region, as well as significantly higher numbers of BrdU-, and DCX-positive cells in the subventricular region in the MFG-E8-treated group than in the vehicle group. This indicates that MFG-E8 reduces inflammation, promotes angiogenesis, and increases neurogenesis in the ischemia model.

MFG-E8 has an important anti-inflammatory effect that is predicted to be effective in reducing secondary neuronal injury in acute cerebral infarction. Studies have demonstrated that reduced MFG-E8 expression is associated with increased inflammatory response. For instance, MFG-E8 expression was found to be considerably reduced in peritoneal macrophages and RAW264.7 macrophages in which an inflammatory response had been induced by lipopolysaccharide (LPS) treatment ${ }^{18,19)}$; the same results were observed in an in vivo animal model of sepsis, and MFG-E8 mRNA expression in the spleen of septic mice decreased after LPS treatment but increased when LPS was administered together with an LPS antagonist, polymyxin- $\mathrm{B}^{24}$. In addition, accumulating evidence indicates that increased MFG-E8 inhibits inflammatory responses. For instance, in a co-culture of macrophages and apoptotic cells, MFG-E8 was found to suppress the LPS-mediated inflammatory response by reducing mitogen-activated protein kinases and NF- $\mathrm{BB}$ signaling ${ }^{11,12,17)}$; moreover, during the LPS-tolllike receptor 4 (TLR4) inflammatory response induced by the phagocytosis of apoptotic cells, MFG-E8 showed an antiinflammatory effect, and in sepsis and ischemia/reperfusion injury mouse models, MFG-E8 inhibited the LPS-TLR4-mediated inflammatory response by reducing production of inflammatory cytokines ${ }^{14,20,21)}$. Collectively, MFG-E8 inhibits the macrophage-mediated inflammatory response by modulating phagocytosis of apoptotic cells via the LPS-TLR4 pathway, and the activity of intracellular signaling molecules unrelated to phagocytosis. Therefore, we hypothesized that MFG-E8 reduces secondary neuronal injury caused by inflammatory cytokine activation. In our study, a significant reduction in Iba-1-positive cells was found in the MFG-E8treated group than in the vehicle group, indicating that MFG-E8 suppresses the inflammatory response in the subacute cerebral infarction rat model.

Additionally, our study demonstrated improved neurobehavioral functional outcomes, as well as increased RECA-1-, BrdU-, and DCX-positive cell numbers, in the MFG-E8 treated group. These results suggest that the reduction in inflammatory response induced by MFG-E8 treatment is associated with increased angiogenesis and neuronal proliferation in the subacute cerebral infarction model. The findings are consistent with those in studies using a similar experimental model of stroke ${ }^{6,7,10,15,23,25)}$, which indicate the potential of MFG-E8 as a neuroprotective agent to help improve prognosis after acute cerebral infarction.

The present study showed that intravenous MFG-E8 treatment did not reduce the infarct volume but improved functional recovery. In contrast, Cheyuo et al. ${ }^{5)}$ found that intravenous administration of exogenous recombinant human MFGE8 (rhMFG-E8) 1 hour after ischemia reduced the infarct size and improved neurological function at both 24 hours and 48 hours after the onset of cerebral ischemia. In addition, histopathological examination showed that rhMFG-E8 treatment protected neurons against necrosis in the penumbra 24 hours after cerebral ischemia onset.

Moreover, studies have found that inflammatory cytokine secretion peaks 24 hours after cerebral ischemia onset, and intravenously injected MFG-E8 does not affect the infarct volume in permanent cerebral ischemia ${ }^{9}$. Therefore, our findings may be attributed to the fact that MFG-E8 was administered during the subacute stage of cerebral infarction 24 hours after 


\section{MCAO.}

There were several limitations to this study. First, the sample size was small and the number of inflammatory cytokines assessed was limited. Second, more categories of experimental infarction groups could have been used. In this experiment, only a 90-minute MCAO group was used; the addition of a 30-minute or 60-minute MCAO group could enable verification of various potential neuroprotective effects of MFG-E8 according to the severity of cerebral infarction. Finally, evaluation of neurological deficits was based solely on a behavioral assessment.

\section{CONCLUSION}

Our study indicates that intravenous administration of MFG-E8 improves functional recovery and effectively attenuates inflammatory responses, suggesting that MFG-E8 could be used as a neuroprotective and regenerative treatment during the subacute stage of cerebral infarction. The findings suggest that MFG-E8 improves neurological function by suppressing inflammation, and increasing angiogenesis and neuronal proliferation during the subacute stage of cerebral ischemia. Further studies with a larger sample size are needed to confirm our findings.

\section{CONFLICTS OF INTEREST}

No potential conflict of interest relevant to this article was reported.

\section{AUTHOR CONTRIBUTIONS}

Conceptualization : DHP, JHK

Data curation : HYK

Formal analysis : HYK

Funding acquisition : DHP

Methodology : DHP

Project administration : $\mathrm{CH}, \mathrm{DHW}$

Visualization : HYK, JIC

Writing - original draft : JIC

Writing - review \& editing : JIC, DHP

\section{ORCID}

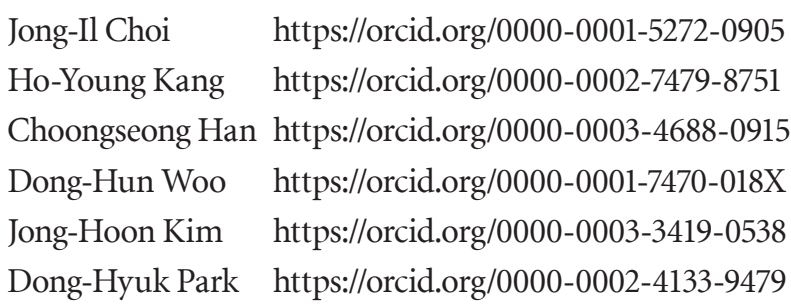

\section{- Acknowledgements}

This study was supported in part by grants from Korea University (K1809681 and K1912651); the Basic Science Research Program through the National Research Foundation of Korea (NRF) funded by the Ministry of Science, ICT \& Future Planning (2013R1A1A2057994); and the Korean Health Technology R\&D Project, Ministry of Health \& Welfare, Republic of Korea (HI12C03370400). The funding bodies did not play any role in the design, collection, analysis, or interpretation of the data; in the writing of the manuscript; or in the decision to submit the manuscript for publication.

\section{References}

1. Barone $F C$ : Ischemic stroke intervention requires mixed cellular protection of the penumbra. Curr Opin Investig Drug $10: 220-223,2009$

2. Borlongan $C V$, Hadman $M$, Sanberg CD, Sanberg PR : Central nervous system entry of peripherally injected umbilical cord blood cells is not required for neuroprotection in stroke. Stroke 35 : 2385-2389, 2004

3. Broughton BR, Reutens DC, Sobey CG : Apoptotic mechanisms after cerebral ischemia. Stroke 40 : e331-e339, 2009

4. Chen J, Li Y, Wang L, Zhang Z, Lu D, Lu M, et al. : Therapeutic benefit of intravenous administration of bone marrow stromal cells after cerebral ischemia in rats. Stroke 32 : 1005-1011, 2001

5. Cheyuo C, Jacob A, Wu R, Zhou M, Qi L, Dong W, et al. : Recombinant human MFG-E8 attenuates cerebral ischemic injury: its role in antiinflammation and anti-apoptosis. Neuropharmacology 62 : 890900, 2012

6. Choi KS, Kim HJ, Do SH, Hwang SJ, Yi HJ : Neuroprotective effects of hydrogen inhalation in an experimental rat intracerebral hemorrhage model. Brain Res Bull 142 : 122-128, 2018

7. Choi TM, Yun M, Lee JK, Park JT, Park MS, Kim HS : Proteomic analysis of a rat cerebral ischemic injury model after human cerebral endothelial cell transplantation. J Korean Neurosurg Soc 59 : 544-550, 2016

8. Collins C, Nehlin JO, Stubbs JD, Kowbel D, Kuo WL, Parry G : Mapping of a newly discovered human gene homologous to the apoptosis 
associated-murine mammary protein, MFG-E8, to chromosome $15 \mathrm{q} 25$. Genomics 39 : 117-118, 1997

9. Emsley HC, Tyrrell PJ : Inflammation and infection in clinical stroke. J Cereb Blood Flow Metab 22 : 1399-1419, 2002

10. Fricker M, Neher JJ, Zhao JW, Théry C, Tolkovsky AM, Brown GC : MFGE8 mediates primary phagocytosis of viable neurons during neuroinflammation. J Neurosci 32 : 2657-2666, 2012

11. Hanayama R, Tanaka M, Miwa K, Shinohara A, Iwamatsu A, Nagata S : Identification of a factor that links apoptotic cells to phagocytes. Nature 417 : 182-187, 2002

12. Henson PM, Bratton DL, Fadok VA : Apoptotic cell removal. Curr Biol 11 : R795-R805, 2001

13. Kim TG, Kwon O, Shin YS, Sung JH, Koh JS, Kim BT : Endovascular treatments performed collaboratively by the Society of Korean Endovascular Neurosurgeons Members : a nationwide multicenter survey. J Korean Neurosurg Soc 62 : 502-518, 2019

14. Lauber K, Keppeler H, Munoz LE, Koppe U, Schröder K, Yamaguchi H, et al. : Milk fat globule-EGF factor 8 mediates the enhancement of apoptotic cell clearance by glucocorticoids. Cell Death Differ 20 : 12301240, 2013

15. Legos JJ, Whitmore RG, Erhardt JA, Parsons AA, Tuma RF, Barone FC : Quantitative changes in interleukin proteins following focal stroke in the rat. Neurosci Lett 282 : 189-192, 2000

16. Lu C, Hua F, Liu L, Ha T, Kalbfleisch J, Schweitzer J, et al. : Scavenger receptor class-A has a central role in cerebral ischemia-reperfusion injury.

J Cereb Blood Flow Metab 30 : 1972-1981, 2010

17. Miksa M, Amin D, Wu R, Jacob A, Zhou M, Dong W, et al. : Maturationinduced down-regulation of MFG-E8 impairs apoptotic cell clearance and enhances endotoxin response. Int J Mol Med 22 : 743-748, 2008

18. Miksa M, Amin D, Wu R, Ravikumar TS, Wang P : Fractalkine-induced MFG-E8 leads to enhanced apoptotic cell clearance by macrophages. Mol Med 13 : 553-560, 2007
19. Miksa M, Wu R, Dong W, Das P, Yang D, Wang P : Dendritic cell-derived exosomes containing milk fat globule epidermal growth factor-factor VIII attenuate proinflammatory responses in sepsis. Shock 25 : 586-593, 2006

20. Miksa M, Wu R, Dong W, Komura H, Amin D, Ji Y, et al. : Immature dendritic cell-derived exosomes rescue septic animals via milk fat globule epidermal growth factor-factor VIII [corrected]. J Immunol 183 : 59835990, 2009

21. Oshima K, Aoki N, Negi M, Kishi M, Kitajima K, Matsuda T : Lactationdependent expression of an mRNA splice variant with an exon for a multiply 0-glycosylated domain of mouse milk fat globule glycoprotein MFG-E8. Biochem Biophys Res Commun 254 : 522-528, 1999

22. Phan TG, Wright PM, Markus R, Howells DW, Davis SM, Donnan GA : Salvaging the ischaemic penumbra: more than just reperfusion? Clin Exp Pharmacol Physiol 29 : 1-10, 2002

23. Savill J, Dransfield I, Gregory C, Haslett C : A blast from the past: clearance of apoptotic cells regulates immune responses. Nat Rev Immunol 2 : 965-975, 2002

24. Schilling M, Besselmann $M$, Leonhard C, Mueller M, Ringelstein EB, Kiefer $\mathrm{R}$ : Microglial activation precedes and predominates over macrophage infiltration in transient focal cerebral ischemia: a study in green fluorescent protein transgenic bone marrow chimeric mice. Exp Neurol $183: 25-33,2003$

25. Silvestre JS, Théry C, Hamard G, Boddaert J, Aguilar B, Delcayre A, et al. : Lactadherin promotes VEGF-dependent neovascularization. Nat Med $11: 499-506,2005$

26. Stubbs JD, Lekutis $C$, Singer KL, Bui A, Yuzuki D, Srinivasan $U$, et al. : cDNA cloning of a mouse mammary epithelial cell surface protein reveals the existence of epidermal growth factor-like domains linked to factor VIII-like sequences. Proc Natl Acad Sci U S A 87 : 8417-8421, 1990 